\title{
Inhibition of matrix metalloproteinase 2 maturation and HT1080 invasiveness by a synthetic furin inhibitor
}

\author{
Erik Maquoi ${ }^{\mathrm{a}}$, Agnès Noël ${ }^{\mathrm{a}}$, Francis Frankenne ${ }^{\mathrm{a}}$, Herbert Angliker ${ }^{\mathrm{b}}$, Gillian Murphy ${ }^{\mathrm{c}}$, Jean-Michel Foidart ${ }^{\mathrm{a}}$ \\ ${ }^{a}$ Laboratoire de Biologie des Tumeurs et du Développement, Université de Liège, Tour de Pathologie (B23), Sart Tilman, B-4000 Liège, \\ Belgium \\ ${ }^{\mathrm{b}}$ Friedrich Institut, Basel, Switzerland \\ 'School of Biological Sciences, University of East Anglia, Norwich, UK
}

\begin{abstract}
The close correlation observed between matrix metalloproteinase 2 (MMP-2) activation and metastatic progression in various tumors suggests that MMP-2 is a 'master switch' triggering tumor spread. Recently, membrane type 1 MMP (MT1-MMP) was identified as a potential physiological activator of MMP-2. Like all other MMPs, MT1-MMP possesses a pro-domain which must be removed for the enzyme to acquire its catalytic potential. The presence of a typical recognition motif (RXKR) for the furin-like convertases at the end of its prodomain suggests a potential role for these proteinases in MT1-MMP processing. In order to evaluate the implication of furin in pro-MT1-MMP processing, we treated HT1080 cells with a synthetic furin inhibitor and monitored their ability to activate pro-MMP-2 as well as their invasive potential. Our results demonstrated that the furin inhibitor decreased pro-MT1-MMP processing as well as pro-MMP-2 activation and cell invasiveness. Therefore, our data bring further evidence that furin is a key factor in the maturation of MMPs associated with the invasive and metastatic potential of tumor cells.
\end{abstract}

Key words: membrane type 1 matrix metalloproteinase ; matrix metalloproteinase 2 ; furin ; Activation ; tumor cell ; invasion

Abbreviations: BM, basement membrane ; ECM, extracellular matrix ; mAb, monoclonal antibody ; MMP, matrix metalloproteinase ; MT1-MMP, membrane type 1 MMP ; TIMP, tissue inhibitor of metalloproteinase ; TPA, 12-O-tetradecanoyl-phorbol 13-acetate

\section{Introduction}

Tumor invasion and metastasis represent a multistep process that depends on the activity of many proteins [1]. Proteolytic degradation of the extracellular matrix (ECM) components is a central event of this process. More specifically, the ability to penetrate the basement membrane (BM) is associated with an increased potential for metastasis. Indeed, BM are present at key points in the metastatic cascade: escape from the primary tumor in epithelial malignancies, intravasa-tion and extravasation during hematogenous dissemination as well as perineural and muscular invasion require BM breaching [2].

Several classes of proteinases, including serine proteinases, cysteine proteinases and matrix metalloproteinases (MMPs), have been implicated in tumor cell invasion process [3]. Among these different proteinases, MMPs appear to be primarily responsible for much of the ECM degradation observed during invasive processes. MMP-2 (also known as gelatinase A or 72-kDa type IV collagenase) and MMP-9 (also known as gelatinase B or 92-kDa type IV collagenase) has been frequently associated with the invasive and metastatic potential of tumor cells [1,4-6]. In general, MMPs are secreted in a latent form (pro-MMP) requiring proteolytic processing in the $\mathrm{N}$-terminal region in order to become catalytically active [7]. This proteolytic maturation is essentially mediated by serine proteinases (including plasmin and trypsin) or some activated MMPs (stromelysin). Unlike the other MMPs, pro-MMP-2 appears refractive to these 'classical' activators [8,9]. Several reports have emphasized the involvement of a plasma membrane-dependent mechanism of activation for pro-MMP-2 [9-12]. In this context, Sato and coworkers [13] have recently discovered MT1-MMP (for membrane type 1 MMP/MMP-14), a novel MMP possessing a hydrophobic C-terminal transmembrane domain overexpression of which induces pro-MMP-2 maturation [13-16], indicating that MT1-MMP is a physiological activator of MMP-2. In addition to its activating capacity, MT1-MMP also acts as a receptor for MMP-2 [17], focusing MMP-2 activity to the vicinity of the cell surface, thus resulting in an increased rate of substrate cleavage [18]. Collectively, these data strongly suggest a key involvement of MT1-MMP during invasive processes.

A peculiar characteristic of MT1-MMP (also shared by stromelysin-3/MMP-11) is the presence of a 11-amino acid insertion in the propeptide at the junction with the catalytic domain of the latent enzyme. The insertion contains a conserved RXKR recognition motif for a group of transmembrane serine proteinases of the subtilisin family termed furin/PACE/kex-2-like proteinases [19], suggesting a potential involvement of these proteinases in 
pro-MT1-MMP processing [13]. In agreement with this proposal, different reports brought evidence that furin was able to cleave transmembrane-deletion mutants of MT1-MMP [20-22]. In contrast with these soluble mutants, wild type enzyme appeared resistant to furin activation. In addition, pro-MT1-MMP induced pro-MMP2 activation, suggesting that furin-induced processing of MT1-MMP is not a prerequisite for pro-MMP-2 activation [20].

In an attempt to clarify the putative implication of furin in pro-MT1-MMP processing, we treated HT1080 cells with a synthetic furin inhibitor and monitored the ability of these cells to activate pro-MMP-2 as well as their invasive potential. Our results demonstrate that the furin inhibitor reduces the level of mature MT1-MMP, which is paralleled by a decrease in pro-MMP-2 activation as well as in cell invasiveness.

Therefore, our data support the concept that pro-MT1-MMP is processed in vivo via a furin-dependent mechanism.

\section{Materials and methods}

\subsection{Cell culture}

HT1080 human fibrosarcoma cells were maintained in Dulbecco's modified Eagle's medium (DMEM) supplemented with $10 \%$ (v/v) fetal calf serum (FCS), penicillin-streptomycin (100 IU/ml-100 $\mu \mathrm{g} / \mathrm{ml}$ ), $2 \mathrm{mM}$ glutamine and $10 \mathrm{mM}$ HEPES buffer at $37^{\circ} \mathrm{C}$ in a humid atmosphere $\left(5 \% \mathrm{CO}_{2}\right.$ and $95 \%$ air). All culture reagents were purchased from Gibco-BRL.

\subsection{Coating of culture wells with type IV collagen}

96-well plates (Falcon, Becton-Dickinson) and 6.5-mm polycarbonate filters ( $8 \mu \mathrm{m}$ pore size) of Transwell cell culture chamber inserts (Costar) were coated with $100 \mu$ of type IV collagen purified from human placenta (kindly provided by Dr. H.W. Krell, Boehringer Mannheim, Penzberg, Germany) diluted at $200 \mu \mathrm{g} / \mathrm{ml}$ in phosphate-buffered saline (PBS). Coats were left to air-dry overnight in a laminar flow hood under UV light. Uniformity of the coating was assessed by Coomassie blue staining. Before use, coated plates and inserts were washed twice with water and incubated for $1 \mathrm{~h}$ in serum-free DMEM at $37^{\circ} \mathrm{C}$.

\subsection{Preparation of conditioned medium (CM) and cell lysates (CL)}

HT1080 cells exponentially growing in T-75 flasks (Nunc, Costar) were collected by trypsin-EDTA treatment, washed with serum-containing medium and allowed to recover from trypsinization for at least $30 \mathrm{~min}$ at $37^{\circ} \mathrm{C}$. Cells were then washed twice in serum-free DMEM and diluted in the same medium supplemented with $0.1 \%$ BSA (fraction V, Sigma) to a density of $3 \times 10^{5}$ cells $/ \mathrm{ml}$. This suspension was plated in type IV collagen-coated 96-well plates (see above), $200 \mu \mathrm{l} /$ well, and cultured for $48 \mathrm{~h}$ in the presence of the synthetic furin inhibitor Dec-Arg-Val-Lys-Arg- $\mathrm{CH}_{2} \mathrm{Cl}$ [23] at concentrations varying from 0 to $100 \mu \mathrm{M}$. The resultant culture supernatants were collected and stored at $-20^{\circ} \mathrm{C}$. Cell monolayers were washed once with $100 \mu \mathrm{l}$ serum-free DMEM and extracted in $50 \mu \mathrm{l}$ of Ripa buffer (50 mM Tris-HCl, pH 7.4, $150 \mathrm{mM} \mathrm{NaCl,} \mathrm{1 \%} \mathrm{Nonidet} \mathrm{P40,} \mathrm{1 \%}$ Triton X-100, 1\% Na-deoxycholate, $0.1 \%$ sodium dodecyl sulfate [SDS], $5 \mathrm{mM}$ iodoacetamide, $2 \mathrm{mM}$ phenylmethylsulfonyl fluoride) for $2 \mathrm{~h}$ at $4^{\circ} \mathrm{C}$.

\subsection{Gelatin zymography}

Analysis of gelatinolytic activities in CM and CL was performed by gelatin zymography. Samples (10 $\mu$ l) of CM and CL were mixed with an equal volume of sample buffer $(62.5 \mathrm{mM}$ Tris-HCl, $\mathrm{pH} 6.8,2 \%$ SDS, $10 \%$ glycerol, $0.1 \%$ bromophenol blue) and subjected to electrophoresis without boiling on $10 \%$ acrylamide gels containing $0.1 \%$ gelatin. Gels were run at $10 \mathrm{~mA}$, washed in $2 \%$ Triton X-100 for $1 \mathrm{~h}$ and incubated at $37^{\circ} \mathrm{C}$ for $16 \mathrm{~h}$ in activation buffer ( $50 \mathrm{mM}$ Tris-HCl, pH 7.4, $200 \mathrm{mM} \mathrm{NaCl}, 5 \mathrm{mM} \mathrm{CaCl}_{2}$ and $0.02 \% \mathrm{NaN}_{3}$ ). After staining with Coomassie brilliant blue R-250, the gelatinolytic activities were detected as clear bands against the blue background. The relative proportions of the gelatinolytic bands corresponding to the different forms of gelatinase A were evaluated by scanning densitometry using a GS-700 Imaging Densitometer (Bio-Rad, Hercules, CA) equipped with Molecular Analyst software. Results are expressed as relative percentages of total gelatinase A activity.

\subsection{Immunoblotting assay for MT1-MMP}

For immunoblotting, samples of CL were mixed with an equal volume of sample buffer with reducing agent (700 mM 2-mercaptoethanol), boiled for $5 \mathrm{~min}$, and subjected to electrophoresis on $8 \%$ polyacrylamide gels. The proteins were electrically transferred to nitrocellulose membranes (Hybond-C extra, Amersham) using a semidry blotting (Bio-Rad). The membranes were blocked with PBS containing 2\% (w/v) BSA (fraction V, Across) and $0.1 \%(\mathrm{v} / \mathrm{v})$ Tween 20 (PBS-BSA-Tween) at room temperature for $60 \mathrm{~min}$ and then probed for $16 \mathrm{~h}$ at $4^{\circ} \mathrm{C}$ with two distinct monoclonal antibodies (mAbs): mAb 1E12 directed against the N-terminal pro-domain of MT1-MMP and mAb 2D7 against the hemopexin-like domain of MT1-MMP (kindly provided by Dr. P. Basset, 
IGBMC, Illkirch, France). The final concentrations of both mAbs were 5 and $20 \mu \mathrm{g} / \mathrm{ml}$, respectively, in PBSBSA-Tween. After extensive washing with PBS containing $0.1 \%$ Tween 20 , the membranes were reprobed with peroxidase-conjugated goat anti-mouse IgG (Dako, Denmark) diluted 1000-fold in PBS-BSA-Tween for 30 min at room temperature and developed with ECL Western blotting detection reagent (Amersham).

\subsection{Invasion assay}

The potential influence of the furin inhibitor $(100 \mu \mathrm{M})$ on HT1080 cell invasion and migration was assayed using Transwell cell culture inserts (Costar). For chemoinvasion assays, type IV collagen-coated membranes (see above) were used whereas uncoated membranes were employed for chemotaxis assays. Lower wells of the chambers contained DMEM supplemented with 20\% FCS and 1\% BSA (fraction V, ICN) as chemoattractant. The upper wells were seeded with $6 \times 10^{4}$ cells prepared as described above. Chambers were subsequently incubated for $48 \mathrm{~h}$ (in order to maintain a chemotactic gradient, media from both lower and upper wells were renewed after $24 \mathrm{~h}$ ) and $4 \mathrm{~h}$ (for chemoinvasion and chemotaxis assays, respectively), in a humid atmosphere at $37^{\circ} \mathrm{C}$. After incubation, filters were removed from the chambers, fixed in $4 \%$ paraformaldehyde for $15 \mathrm{~min}$, permeabilized for $10 \mathrm{~min}$ in methanol $\left(-20^{\circ} \mathrm{C}\right)$ and stained with giemsa $(4 \%)$. The upper surface of the filters was scrapped with a cotton swab and cells having reached the lower surface of the filters were visually counted using a light microscope (Vanox AH3, Olympus) at 400-fold magnification. At least 20 random fields were counted per filter. Each experiment was repeated twice.

\subsection{Cytotoxicity assay}

In order to assess the potential influence of the furin inhibitor on cell metabolism and/or proliferation, cells were plated on type IV collagen-coated 96-well plates in the same culture conditions as described for the preparation of CM (see above) and incubated for $48 \mathrm{~h}$ in the presence of different concentrations of the inhibitor (medium renewal occurred after $24 \mathrm{~h}$ ). Media were subsequently replaced by $100 \mu \mathrm{l}$ of DMEM supplemented with $10 \%$ WST-1 (Boehringer Mannheim) and plates were incubated for $2 \mathrm{~h}$ at $37^{\circ} \mathrm{C}$. Cellular metabolism was determined by measuring the absorbance of the medium at 450 ran (with a reference filter of 595 ran) on a microtiter plate reader (Multiscan MS, Labsystems, Finland). At the concentrations tested (0-100 $\mu \mathrm{M})$, the synthetic furin inhibitor did not significantly affect cellular metabolism and/or proliferation (data not shown).

\subsection{Statistical analysis}

Statistical analysis of the results was performed using Student's $t$-test. Differences were considered as statistically significant for $P$ values lower than 0.05 .

\section{Results}

\subsection{Effect of the synthetic furin inhibitor on MMP-2 activation by HT1080 cells}

When conditioned medium of HT1080 cells cultured for $48 \mathrm{~h}$ on type IV collagen was analyzed by gelatin zymography, four major gelatinolytic bands of $92 \mathrm{kDa}, 66 \mathrm{kDa}, 62 \mathrm{kDa}$ and $59 \mathrm{kDa}$ were detected (Fig. 1A, lane 1). These bands corresponded to the inactive proforms of MMP-9 (92 kDa) and MMP-2 (66 kDa), as well as the processed forms of MMP-2: the 62-kDa intermediate and the fully active 59-kDa species. Addition of increasing concentrations of the synthetic furin inhibitor (see Section 2) for $48 \mathrm{~h}$ resulted in a dose-dependent reduction of the secretion of both intermediate (62-kDa) and mature (59-kDa) MMP-2 forms, concurrently with a gradual increase in pro-MMP-2 level (Fig. 1A,B). It is worth noting that even at the highest concentration $(100 \mu \mathrm{M})$ the inhibitor failed to completely suppress pro-MMP-2 processing (Fig. 1A, lane 5).

\subsection{MT1-MMP processing is modulated by the furin inhibitor}

To further explore if the drastic reduction of pro-MMP-2 activation observed in the presence of the furin inhibitor was correlated with a decreased level of active MT1-MMP, we analyzed the pattern of produced MT1MMP. Cell lysates from HT1080 cells, treated or not for $48 \mathrm{~h}$ with the highest concentration of the furin inhibitor $(100 \mu \mathrm{M})$, were analyzed by Western blotting using two distinct mAbs directed against the pro- (mAb 1E12) and the hemopexin-like (mAb 2D7) domains. Lysates from COS cells transiently transfected to overexpress MT1MMP were used as a positive control [24]. Lysates of untreated HT1080 cells contained four different immunoreactive proteins: a doublet composed of 66-kDa and 63-kDa species, both recognized by mAb 1E12 (Fig. 2A, lane 1). This doublet co-migrated with those of MT1-MMP-transfected COS-1 cell lysates (data not shown). In addition, a 60-kDa and a faint 43-kDa protein were revealed by mAb 2D7 (Fig. 2B, lane 1).

Although treatment with the furin inhibitor did not cause any alteration of the level of the 63-kDa protein (Fig. 2A, lane 2), the amounts of $60-\mathrm{kDa}$ and $43-\mathrm{kDa}$ proteins were significantly reduced, the latter being nearly undetectable (it should be noted that the potential presence of already matured MT1-MMP species at the cell surface before the addition of the furin inhibitor most probably underestimates its effectiveness). In contrast, the expression of the 66-kDa form was noticeably increased (Fig. 2B, lane 2). These data demonstrate that, in the 
experimental conditions used, treatment of cells with a synthetic furin inhibitor results in an accumulation of unprocessed forms, which strongly suggests a decreased pro-MT1-MMP maturation.

Fig. 1. Activation of pro-MMP-2 by HT1080 cells treated with increasing concentrations of furin inhibitor. Cells were cultured for $48 \mathrm{~h}$ on type IV collagen in the absence (lane 1) or presence of furin inhibitor: $10 \mu \mathrm{M}$ (lane 2), $25 \mu \mathrm{M}$ (lane 3), $50 \mu \mathrm{M}$ (lane 4) and $100 \mu \mathrm{M}$ (lane 5). A: Zymographic analysis of conditioned medium. B: Quantification of pro-MMP-2 activation by scanning densitometry of the gel depicted in $A$. The relative proportions of MMP-2 pro-(66-kDa; hatched bar), intermediate (62-kDa; white bar), and mature (59-kDa; black bar) forms is expressed in percent of total MMP-2.

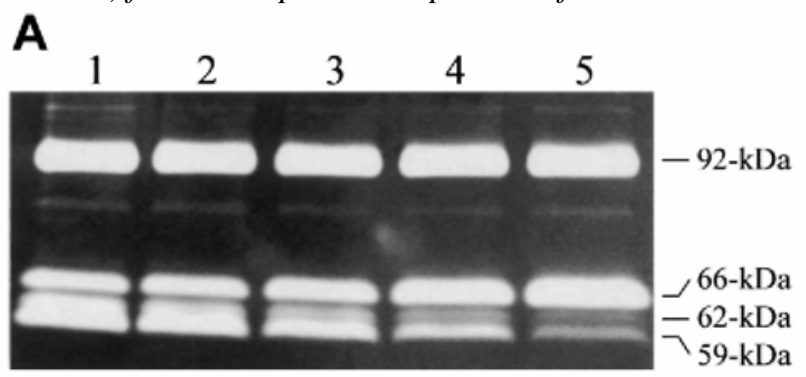

\section{B}

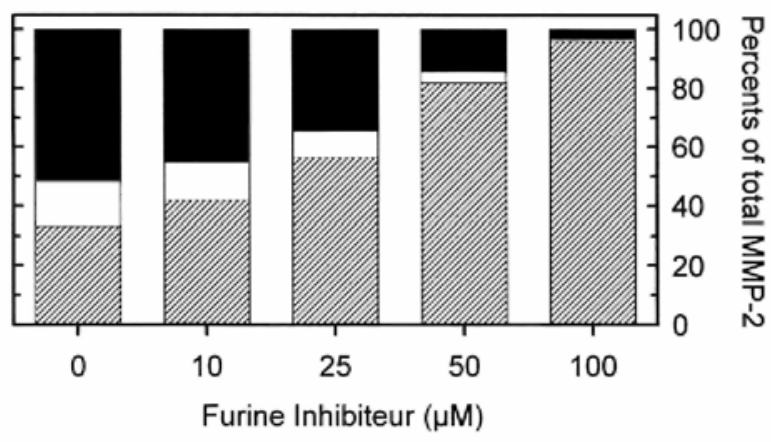

Fig. 2. Influence of furin inhibitor on MT1-MMP processing. HT1080 cells were cultured for 48 h on type IV collagen in the absence (lane 1) or presence (lane 2) of furin inhibitor $(100 \mu \mathrm{M})$. Cell extracts were analyzed by Western blotting using mAb $1 E 12$ directed against the pro-domain of MT1-MMP (A) and mAb $2 D 7$ directed against the hemopexin-like domain of MT1-MMP (B).

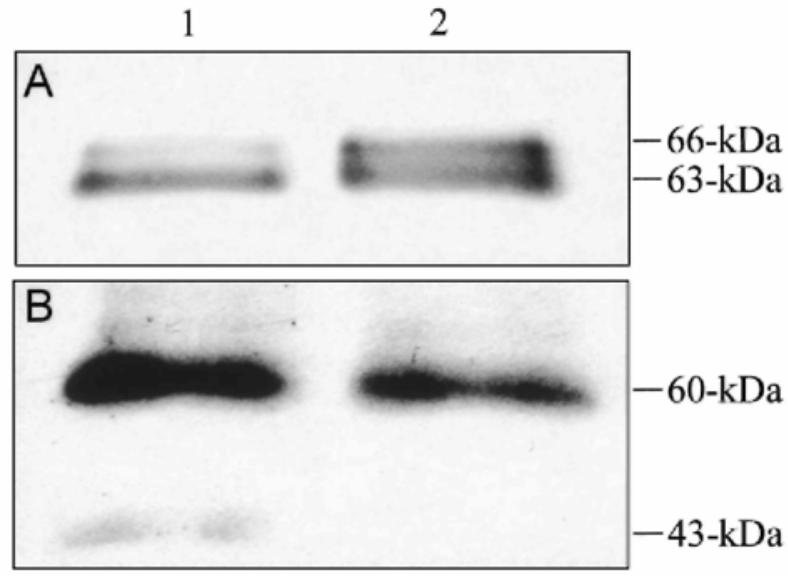

\subsection{Furin inhibitor reduces HT1080 invasiveness}

To determine whether the presence of activated MT1-MMP contributes to the invasive behavior of HT1080 cells, we measured the ability of cells treated or not with the furin inhibitor to breach and to invade through type IV collagen-coated Transwell cell culture inserts. In the absence of furin inhibitor, cells were able to process proMMP-2, which was subsequently released in the culture medium (Fig. 3B, Control) or bound to type IV collagen and/or cell surface receptors (Fig. 3C, Control). In these conditions, the cells readily dissolve the collagen layer 
and migrate through the filter (Fig. 3A, Control). Addition of the furin inhibitor dramatically reduced the amount of both secreted and bound active MMP-2 (Fig. 3B,C, Furin Inhibitor), and decreased by 5-fold the cell invasiveness (Fig. 3A, Furin Inhibitor; $P=0.0003$ ). In contrast, cell migration through uncoated filters was not significantly affected $(P=0.51)$ (as measured in the chemotaxis assay, Fig. 3A). The degree of invasion inhibition obtained here with the furin inhibitor was similar to those observed with recombinant TIMP-2 or synthetic broad-spectrum MMP inhibitors (such as GI129471 or BB-94), both treatments preventing pro-MMP-2 activation (manuscript in preparation).

Fig. 3. Furin inhibitor reduces HT1080 invasiveness. A: HT1080 chemoinvasion (hatched bar) and chemotaxis (white bar) were evaluated in Transwell cell culture inserts coated or not with type IV collagen, respectively. HT1080 cells were seeded in the absence (control) or presence of furin inhibitor $(100 \mu M)$ and the number of cells that have migrated was determined by visually counting the number of cells present on the lower side of the filters (see Section 2). Results are expressed as percentages of the migration of control cells. In order to monitor the activation status of MMP-2 during the invasion assay, conditioned media (B) and cell extracts (C) from the invasion experiments were collected and analyzed by gelatin zymography. The relative proportions of the pro(66-kDa; white bar) and activated (62-kDa and 59-kDa; hatched bar) forms of MMP-2 were determined from scanning densitometry data and are expressed as percent of total MMP-2.
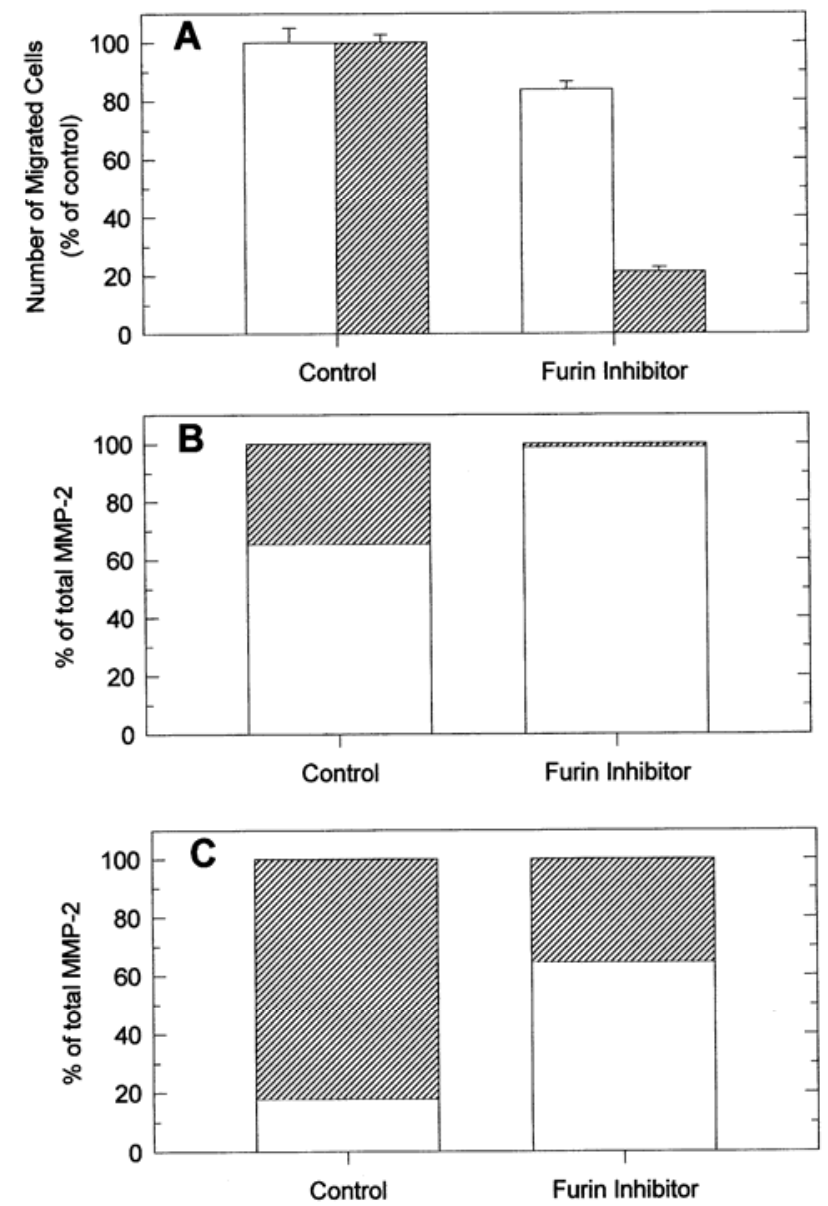

\section{Discussion}

So far, MMP-2 overexpression by tumor cells and/or their associated stroma has been considered a key event associated with tumor progression and metastasis (see [25], for review). Nevertheless, even if the overproduction of the pro-enzyme appears necessary for the acquisition of an invasive phenotype, it is not by itself a sufficient criterion [26]. Indeed, most MMPs are produced as latent zymogens in which catalytic activity is tightly regulated. Regulation involves both the control of zymogen activation and the inhibition of the active enzyme by specific inhibitors such as TIMPs. In this context, the close correlation observed between MMP-2 activation and metastatic progression in various tumors [27-29] suggests that MMP-2 activator is a 'master switch' triggering tumor spread. Recently, Sato and coworkers identified MT1-MMP as a potential physiological activator of MMP-2 [13]. Accordingly, expression of this membrane-associated MT1-MMP has been shown to mediate pro- 
MMP-2 activation as well as to stimulate in vitro invasiveness of different cell lines. Moreover, the expression of elevated levels of MT1-MMP in tumor samples in which MMP-2 is activated suggests that MT1-MMP is actually involved in MMP-2 processing in vivo [17,30-33]. Like all other known MMPs, MT1-MMP possesses a pro-domain that confers latency which must be removed for the enzyme to acquire its catalytic potential. The presence of a typical recognition motif (RXKR) for furin-like serine proteinases between the pro- and catalytic domains of MT1-MMP would suggest a potential role for these proteinases in MT1-MMP maturation [13]. However, by using transfected cell lines overexpressing different MT1-MMP forms, some authors showed that only soluble (obtained by deletion of the transmembrane region of the protein) and not membrane-anchored forms of MT1-MMP were efficiently processed by furin [20-22]. In contrast to soluble pro-MT1-MMP, which was unable to activate MMP-2 before being processed by furin [21], membrane-bound pro-MT1-MMP efficiently activated MMP-2 [20]. These data contrasted with the isolation by Strongin and coworkers of an activated form of membrane-associated MT1-MMP from TPA-stimulated HT1080 cells that readily activated MMP-2 [10]. These controversial observations prompted us to examine the potential role(s) of furin in MT1MMP activation in an intact cell system. For this purpose, HT1080 cells were treated with a synthetic furin inhibitor [23] previously shown to block the furin-dependent processing of pro-stromelysin-3 [34].

Western blot analysis of cell lysates obtained from HT1080 cells cultured on type IV collagen revealed the presence of different immunoreactive MT1-MMP species corresponding to a 63-kDa proform [13,20] as well as a 60-kDa protein previously identified as activated MT1-MMP [10]. Additional bands of 66 and $43 \mathrm{kDa}$ were also detected. The 43-kDa form has been proposed to be a proteolytic fragment of either the 63-kDa or the 60$\mathrm{kDa}$ form. It is worth noting that the occurrence of this 43-kDa species has been correlated with pro-MMP-2 activation. However, whether this species actively participates in MMP-2 processing remains unknown [35]. Consistent with this correlation, we showed that our cells efficiently activated pro-MMP-2 through a typical twostep process, previously shown to be MT1-MMP-dependent $[13,15]$. In the presence of the furin inhibitor, the capacity of HT1080 cells to process pro-MMP-2 was dramatically impaired, as judged by the almost complete disappearance of both intermediate and mature forms of MMP-2. Our immunoblotting data obtained from the corresponding cell lysates revealed that the inhibition of furin clearly reduced the processing of pro-MTl-MMP into both the $60-\mathrm{kDa}$ and $43-\mathrm{kDa}$ forms, the latter being almost undetectable. This observation further supports the putative relationship that could link pro-MMP-2 activation and the presence of the 43-kDa form of MT1MMP in cell lysates. While the inhibition of furin did not alter the amount of produced 63-kDa pro-MTl-MMP, a significant increase of the 66-kDa form was noted. The exact nature of this form is actually unknown. However, it can be speculated that this higher molecular weight species represents a post-translationally modified form of the 63-kDa pro-MT1-MMP which is subsequently processed by furin during its secretion. A similar process has been previously described during stromelysin-3 maturation [34]. Altogether, these findings indicate that furin or furin-like convertases are indeed involved in the activation mechanism of pro-MT1-MMP. Moreover, even if the furin inhibitor failed to completely prevent the appearance of activated MT1-MMP species, the maturation of pro-MMP-2 was dramatically inhibited, thus confirming that MMP-2 processing requires activated MT1-MMP.

In order to further investigate the influence of the inhibition of furin on cell invasiveness, chemoinvasion assays through type IV collagen-coated filters were performed. Our results clearly demonstrate that the ability of HT1080 cells to degrade and subsequently invade through type IV collagen was greatly reduced by the furin inhibitor. This impaired invasiveness is most probably the consequence of the reduced amount of active MMP-2. In addition, activated MT1-MMP also has the capacity to directly digest several components of the ECM [21,36], thus facilitating cell invasion.

Taken together, these data bring further evidence that furin is a key factor in the maturation of MMPs (MT1MMP and MMP-2) associated with the invasive and metastatic potential of tumor cells.

\section{Acknowledgements}

The authors would like to thank J.P. Cherami-Bien for performing invasion assays, Dr. P. Basset (IGBMC, Illkirch, France) for providing anti-MT1-MMP monoclonal antibodies and Dr. E.W. Thompson (Lombardi Cancer Center, Georgetown University Medical Center, Washington, DC) for providing MT1-MMP-transfected COS-1 cells lysates. This work was supported by grants from the Communauté Française de Belgique (Actions de Recherche Concertées 93/98-171 and 95/00-191), the CGER-Assurances 1996/ 1999, the Association contre le Cancer, the Association Sportive contre le Cancer, the Loterie Nationale (9.4573.96F), the Fonds de la Recherche Scientifique Médicale (FRSM) (3.4573.95), the Fonds d'Investissement de Recherche Scientifique 1997-CHU Liège, the Centre Anticancéreux près de l'Université de Liège and the Fondation Léon Frédéricq, University of Liège, Liège, all in Belgium, the General RE-Luxembourg, the Commission of European Communities (Concerted European Action Biotech BIO4-CT96-0464) and industry (Boehringer Mannheim GmbH, Germany). 
Published in: FEBS Letters (1998), vol. 424, iss. 3, pp. 262-266.

Status: Postprint (Author's version)

\section{References}

[1] Liotta, L.A., Steeg, P. and Stetler-Stevenson, W.G. (1991) Cell 64, 327-336.

[2] Stetler-Stevenson, W.G., Liotta, L.A. and Kleiner, D.E. (1993) FASEB J. 7, 1434-1441.

[3] Duffy, M.J. (1992) Clin. Exp. Metastasis 10, 145-155.

[4] Tryggvason, K., Höyhtyä, M. and Salo, T. (1987) Biochim. Biophys. Acta 907, 191-217.

[5] Collier, I.E., Wilhelm, S.M., Eisen, A.Z., Marmer, B.L., Grant, G.A., Seltzer, J.L., Kronberger, A., He, C., Bauer, E.A. and Goldberg, G.I. (1988) J. Biol. Chem. 263, 6579-6587.

[6] Levy, A.T., Cioce, V., Sobel, M.E., Garbisa, S., Grigoni, W.F., Liotta, L.A. and Stetler-Stevenson, W.G. (1991) Cancer Res. 51, $439-444$.

[7] Nagase, H. (1997) J. Biol. Chem. 378, 151-160.

[8] Okada, Y., Morodomi, T., Enghild, J.J., Suzuki, K., Yasui, A., Nakanishi, I., Salvesen, G. and Nagase, H. (1990) Eur. J. Biochem. 194, 721-730.

[9] Strongin, A.Y., Marmer, B.L., Grant, G.A. and Goldberg, G.I. (1993) J. Biol. Chem. 268, 14033-14039.

[10] Strongin, A.Y., Collier, I., Bannikov, G., Marmer, B.L., Grant, G.A. and Goldberg, G.I. (1995) J. Biol. Chem. 270, 5331-5338.

[11] Brown, P.D., Bloxidge, R.E., Anderson, E. and Howell, A. (1993) Clin. Exp. Metastasis 11, $183-189$.

[12] Ward, R.V., Atkinson, S.J., Slocombe, P.M., Docherty, A.J.P., Reynolds, J.J. and Murphy, G. (1991) Biochim. Biophys. Acta 1079, 242-246.

[13] Sato, H., Takino, T., Okada, Y., Cao, J., Shinagawa, A., Yamamoto, E. and Seiki, M. (1994) Nature 370, 61-65.

[14] Cao, J., Sato, H., Takino, T. and Seiki, M. (1995) J. Biol. Chem. 270, 801-805.

[15] Atkinson, S.J., Crabbe, T., Cowell, S., Ward, R.V., Butler, M.J., Sato, H., Seiki, M., Reynolds, J.J. and Murphy, G. (1995) J. Biol. Chem. 270, 30479-30485.

[16] Sato, H., Takino, T., Kinoshita, T., Imai, K., Okada, Y., Stetler-Stevenson, W.G. and Seiki, M. (1996) FEBS Lett. 385, 238-240.

[17] Sato, H. and Seiki, M. (1996) J. Biochem. 119, 209-215.

[18] Young, T.N., Pizzo, S.V. and Stack, M.S. (1995) J. Biol. Chem. 270, 999-1002.

[19] Seidah, N.G., Chrétien, M. and Day, R. (1994) Biochimie 76, 197-209.

[20] Cao, J., Rehemtulla, A., Bahou, W. and Zucker, S. (1996) J. Biol. Chem. 271, 30174-30180.

[21] Pei, D. and Weiss, S.J. (1996) J. Biol. Chem. 271, 9135-9140.

[22] Imai, K., Ohuchi, E., Aoki, T., Nomura, H., Fujii, Y., Sato, H., Seiki, M. and Okada, Y. (1996) Cancer Res. 56, $2707-2710$.

[23] Angliker, H., Wikstrom, P., Shaw, E., Brenner, C. and Fuller, R.S. (1993) Biochem. J. 293, 75-81.

[24] Pulyaeva, H., Bueno, J., Polette, M., Birembaut, P., Sato, H., Seiki, M. and Thompson, E.W. (1997) Clin. Exp. Metastasis 15, 111-120.

[25] Yu, A.E., Hewitt, R.E., Kleiner, D.E. and Stetler-Stevenson, W.G. (1996) Biochem. Cell. Biol. 74, 823-831.

[26] Stetler-Stevenson, W.G. (1995) Invasion Metastasis 14, 259-268.

[27] Brown, P.D., Bloxidge, R.E., Stuart, N.S.A., Gatter, K.C. and Carmichael, J. (1993) J. Natl. Cancer Inst. 85, 574-578.

[28] Davies, B., Waxman, J., Wasan, H., Abel, P., Williams, G., Krausz, T., Neal, D., Thomas, D., Hanby, A. and Balkwill, F. (1993) Cancer Res. 53, 5365-5369.

[29] Nomura, H., Fujimoto, N., Seiki, M., Mai, M. and Okada, Y. (1996) Int. J. Cancer 69, 9-16.

[30] Nomura, H., Sato, H., Seiki, M., Mai, M. and Okada, Y. (1995) Cancer Res. 55, 3263-3265.

[31] Tokuraku, M., Sato, H., Murakami, S., Okada, Y., Watanabe, Y. and Seiki, M. (1995) Int. J. Cancer 64, $355-359$.

[32] Yamamoto, M., Mohanam, S., Sawaya, R., Fuller, G.N., Seiki, M., Sato, H., Gokaslan, Z.L., Liotta, L.A., Nicolson, G.L. and Rao, J.S. (1996) Cancer Res. 56, 384-392.

[33] Ueno, H., Nakamura, H., Inoue, M., Imai, K., Noguchi, M., Sato, H., Seiki, M. and Okada, Y. (1997) Cancer Res. 57, $2055-2060$.

[34] Santavicca, M., Noël, A., Angliker, H., Stoll, I., Segain, J.P., Anglard, P., Chretien, M., Sato, H. and Seiki, M. (1996) J. Biochem. 119, 209-215.

[35] Lohi, J., Lehti, K., Westermarck, J., Kähäri, V.-M. and Keski-Oja, J. (1996) Eur. J. Biochem. 239, $239-247$.

[36] Ohuchi, E., Imai, K., Fujii, Y., Sato, H., Seiki, M. and Okada, Y. (1997) J. Biol. Chem. 272, 2446-2451. 